\title{
Erratum: Comparison of cellular oscillations driven by noise or deterministic mechanisms under cell-size scaling [Phys. Rev. E 94, 042425 (2016)]
}

\author{
Chong He, Keng-Hwee Chiam, and Lock Yue Chew
}

(Received 24 August 2018; published 14 September 2018)

DOI: 10.1103/PhysRevE.98.039902

In an earlier draft of our paper, we had considered elementary biochemical reactions, such as the binding of two different molecules to form a dimer or the dissociation of a dimer into two separate molecules where each reaction instance only changed the molecule number of the reactants by one at most. While we eventually shifted our attention to composite reactions, we did not update our earlier draft on this aspect. Thus a mistake incurred in the printed version of our paper. The following are the corrections to this mistake. Note that all the changes made in this Erratum do not affect the conclusions of the original paper.

The first correction involves replacing Eq. (1) and the paragraph before it with:

"Our study is presented within the framework of stochastic differential equations. In this framework, a biochemical network is expressed via a tuple: $(\vec{x}, \vec{u}(\vec{x}))$, where $\vec{x}$ is a vector representing the amount of each type of reactant; $\vec{u}(\vec{x})$ is a vector with element $u_{j}$ representing the reaction rate of reaction $j$. In addition, $a_{i j}$ is the change in the number of molecules of reactant $i$ after the occurrence of one instance of reaction $j$. With the system denoted this way, its dynamics can be fully expressed as follows:

$$
d x_{i}=\sum_{j=1}^{r} a_{j i} u_{j}(\vec{x}) d t+\sum_{j=1}^{r} a_{j i} \sqrt{u_{j}(\vec{x})} d B_{i j} . "
$$

Because this error propagates to the other equations, we have to make the following series of replacements:

(a) Change Eqs. (4) and (5) to

$$
v_{i} \equiv \sum_{j=1}^{r} a_{j i} u_{j}(\vec{x}), \quad L_{i} \equiv \sum_{j=1}^{r} a_{j i} \sqrt{u_{j}(\vec{x})} d B_{i j}
$$

(b) change Eq. (7) to

$$
d x_{i}=\sum_{j} a_{j i} u_{j}(\vec{x}) d t
$$

(c) change Eq. (8) to

$$
\hat{\eta}_{c}(\vec{x}, \vec{u}(\vec{x}))=\left(\eta_{c} \vec{x}, \eta_{c} \vec{u}(\vec{x})\right) .
$$

(d) Change the text and equations that are located eight lines below Eq. (8) to "Denoting the system postoperation as $\left(\vec{x}_{c}, \vec{u}_{c}\left(\vec{x}_{c}\right)\right)$ in the reaction chamber of size $V_{c}$, we have

$$
V_{c}=\eta_{c} V, \quad x_{c i}=\eta_{c} x_{i}, \quad u_{c j}\left(\vec{x}_{c}\right)=\eta_{c} u_{j}(\vec{x}), "
$$

(e) Change Eqs. (9) and (10) to

$$
d x_{c i}=\sum_{j}\left(a_{j i} u_{c j}\left(\vec{x}_{c}\right) d t+a_{j i} \sqrt{u_{c j}\left(\vec{x}_{c}\right)} d B_{i j}\right) \quad \Longleftrightarrow d x_{i}=\sum_{j}\left(a_{j i} u_{j}(\vec{x}) d t+\eta_{c}^{-1 / 2} a_{j i} \sqrt{u_{j}(\vec{x})} d B_{i j}\right) .
$$

(f) Change Eq. (12) to

$$
\xi \equiv \frac{\frac{\sum_{j} a_{j i} \sqrt{u_{c j}\left(\vec{x}_{c}\right)}}{\sum_{j} a_{j i} u_{c j}\left(\vec{x}_{c}\right)}}{\frac{\sum_{j} a_{j i} \sqrt{u_{j}(\vec{x})}}{\sum_{j} a_{j i} u_{j}(\vec{x})}}=\eta_{c}^{-1 / 2} .
$$

(g) Change the set of equations after Eq. (25) to

$$
y_{i} \equiv x_{i}-x_{o i},\left.\quad M_{i j} \equiv \frac{\partial}{\partial x_{j}}\left(\sum_{k=1}^{r} a_{k i} u_{k}\right)\right|_{\vec{x}=\vec{x}_{o}}, \quad \sigma_{i k} \equiv a_{k i} \sqrt{u_{k}\left(\vec{x}_{o}\right)},
$$

Finally, we replace Eq. (B2) by the following equations for the purpose of defining $y_{c i}$ and $u_{i}$ :

$$
y_{c i} \equiv x_{c i} \eta_{c}^{-1 / 2} \Gamma_{i}^{-1}, \quad u_{i} \equiv v_{i} \eta_{c}^{1 / 2} \Gamma_{i}^{-1} .
$$

